\title{
Improved Cat Swarm Optimization for Simultaneous Allocation of DSTATCOM and DGs in Distribution Systems
}

\author{
Neeraj Kanwar, Nikhil Gupta, K. R. Niazi, and Anil Swarnkar \\ Department of Electrical Engineering, Malaviya National Institute of Technology Jaipur, Jaipur 302017, India \\ Correspondence should be addressed to Nikhil Gupta; nikhil2007_mnit@yahoo.com
}

Received 9 June 2015; Revised 6 September 2015; Accepted 11 October 2015

Academic Editor: Joydeep Mitra

Copyright ( 2015 Neeraj Kanwar et al. This is an open access article distributed under the Creative Commons Attribution License, which permits unrestricted use, distribution, and reproduction in any medium, provided the original work is properly cited.

\begin{abstract}
This paper addresses a new methodology for the simultaneous optimal allocation of DSTATCOM and DG in radial distribution systems to maximize power loss reduction while maintaining better node voltage profiles under multilevel load profile. Cat Swarm Optimization (CSO) is one of the recently developed powerful swarm intelligence-based optimization techniques that mimics the natural behavior of cats but usually suffers from poor convergence and accuracy while subjected to large dimension problem. Therefore, an Improved CSO (ICSO) technique is proposed to efficiently solve the problem where the seeking mode of CSO is modified to enhance its exploitation potential. In addition, the problem search space is virtually squeezed by suggesting an intelligent search approach which smartly scans the problem search space. Further, the effect of network reconfiguration has also been investigated after optimally placing DSTATCOMs and DGs in the distribution network. The suggested measures enhance the convergence and accuracy of the algorithm without loss of diversity. The proposed method is investigated on 69-bus test distribution system and the application results are very promising for the operation of smart distribution systems.
\end{abstract}

\section{Introduction}

The electric power industries have witnessed many reforms in recent years. The existing distribution systems are moving towards smart distribution systems to achieve larger socioeconomic and other nontangible benefits. The rise of smart grid is a boon not only to society as a whole but also to all who are involved in the electric power industry, its customers, and its stakeholders [1]. Building of such distribution systems requires local generation of reactive and active power using distributed energy resources (DERs) such as Distribution Static Compensators (DSTATCOMs) and Distributed Generations (DGs). DSTATCOM is a power electronic-based synchronous voltage generator capable of providing rapid and uninterrupted capacitive and inductive reactive power supply [2]. Various renewable and nonrenewable DG technologies are available on the market today, such as microturbines, fuel cells, combustion gas turbines, photovoltaic, wind turbines, and combined heat and power [3]. The integration of renewable DG technologies such as photovoltaic and wind turbines is becoming more popular in distribution systems on account of smart grid initiatives and strict environmental laws. These components allow increased efficiency, more reliability, and better quality of electric service. Moreover, they also facilitate effective utilization and life extension of existing distribution system infrastructure [1]. However, optimal placement and sizing of these components are the important issues to extract maximum possible benefits.

The optimal allocation of DSTATCOM and DGs in distribution systems is a highly nonlinear complex combinatorial problem which has to satisfy various equality and inequality constraints. In the recent past, several populationbased metaheuristic techniques such as Genetic Algorithm (GA), Ant Colony Optimization (ACO), Immune Algorithm (IA), Differential Evolution Algorithm (DEA), Firefly Algorithm (FA), Particle Swarm Optimization (PSO), TeachingLearning Based Optimization (TLBO), Artificial Bee Colony (ABC), Harmony Search Algorithm (HSA), and Cuckoo Search Algorithm (CSA) have shown their potential to solve optimal DSTATCOM placement problem [4-7] or optimal DG placement problem [8-12]. However, the simultaneous placement strategy can independently set and control the real and reactive power flow in distribution networks [13]. 
A lot of research work has been carried out to successfully optimize the siting and sizing problems of active and reactive components when allocated separately. However, only a few researchers have attempted simultaneous placement strategy. References [14-17] have shown mutual impact of these components on the performance of distribution networks. Abu-Mouti and El-Hawary [14] employed an ABC algorithm to determine the optimal size of DGs, power factor, and location to minimize power losses. A heuristic approach is suggested by Naik et al. [15] where a node sensitivity analysis is used to identify the candidate DER sites, and their optimal capacities are determined by suggesting heuristic curve fitting technique. Moradi et al. [17] proposed a combined imperialist competitive algorithm- (ICA-) GA method to solve this multiobjective optimization problem. In this method, first ICA is used to find the sites and sizing of DERs and then the operators of GA are employed to further refine these solutions.

The smart grid requires integrated solutions for available distributed resources that reflect their coexistence to achieve higher efficiency through loss minimization and good quality power supply. Distribution networks are reconfigured frequently with changing operating conditions, and it is one of the effective means to improve their performance. The network reconfiguration is a process that alters feeder topological structure by managing the open/close status of sectionalizing and tie-switches under contingencies or normal operating conditions [18]. Changing network topology by reallocating loads from one feeder to another may balance loads among the feeders and decrease the real power losses [19]. Therefore, this is another resource that can be utilized in conjunction with simultaneous placement of DSTATCOM and DG. This approach has possibly not been attempted till date.

Cat Swarm Optimization (CSO) is one of the recently established high performance computational techniques introduced by Chu and Tsai [20]. CSO is inspired by the natural behavior of cats where two major behaviors of the cats are modeled into two submodels: seeking mode and the tracing mode. In the seeking mode, the cat looks around and seeks the next position to move to, whereas, in the tracing mode, the cat tracks some targets [21]. The important property of CSO is that it provides local as well as global search capability simultaneously [22]. It converges better and shows a better performance in finding the global best solution [21]. It has been successfully applied to solve diverse engineering optimization problems such as linear antenna array synthesis [23], deployment of wireless sensors [21], IIR system identification [24], clustering [25], and linear phase FIR filter design [26]. However, the exploration potential of CSO needs to be enhanced while subjected to large dimension problems by reviewing its seeking mode. Further, convergence and accuracy of the algorithm can be improved by suitably placing tentative solutions in the problem search space during the iterative process.

Several researchers $[10,12,15,27]$, and many others, have squeezed the problem search space by restricting the number of candidate locations for placing these devices. They generated a node priority list using certain node sensitivitybased approach and then selecting top few nodes from it as the candidate sites to allocate these devices. This approach drastically reduces the problem search space and also the CPU time incurred. However, the sensitivities are normally calculated for the base case conditions, where no such devices are installed [28]. Furthermore, when selecting only top nodes as the sensitive components, it did not give the true picture of the entire distribution network [29]. Therefore, such approaches are unreliable and thus lead the algorithm to suboptimal solution.

In light of the above discussion, a new Improved CSO(ICSO-) based method is proposed for the simultaneous allocation of DSTATCOM and DGs in radial distribution networks. The objective is to maximize power loss reduction while maintaining a better node voltage profile. The distribution network is reconfigured after the optimal placement of these devices to extract maximum possible benefits. The seeking mode of CSO is modified to enhance exploitation potential of the algorithm. In addition, an intelligent search is proposed to enhance the overall performance of the optimizing tool.

The remainder of the paper is organized as follows: The problem is formulated in Section 2. The description of the standard and proposed CSO algorithm is presented in Sections 3 and 4, respectively. Section 5 deals with simulation results and the analysis of results is discussed in Section 6. Finally, the conclusions drawn from this work are presented in Section 7.

\section{Problem Formulation}

The node voltage profile of distribution systems can be improved by installation of DERs as DGs and DSTATCOMs, network reconfiguration, tap changing transformers, and so forth. The proposed algorithm is installing these components, and then the distribution network is reconfigured. Therefore, a soft voltage constraint is treated as the part of objective function while installing DSTATCOM and DGs, and the solutions are accepted by imposing penalty so long as the voltage constraint violates within prespecified limits. However, a hard voltage constraint is necessary for the system operation. Thus it is employed while reconfiguring the distribution network after optimally placing these components. The amount of voltage profile improvement and the capacities of these components employed are not linearly related. Therefore, the advantage of soft voltage constraint employed in placing DSTATCOMs and DGs is that it results in lesser capacity allocation in the optimal solution. In other words, the whole burden of voltage profile improvement should not be imposed over these distributed components, as the network reconfiguration can successfully improve voltage profiles. Therefore, the objective function $F_{i}$ is formulated to maximize power loss reduction while maintaining a better node voltage profile by proposing a voltage penalty factor approach as defined below:

$$
\operatorname{Max} F_{i}=\mathrm{PF}_{i} \times\left(P_{\text {loss }, b i}-P_{\text {loss }, a i}\right) ; \quad \forall i \in L,
$$




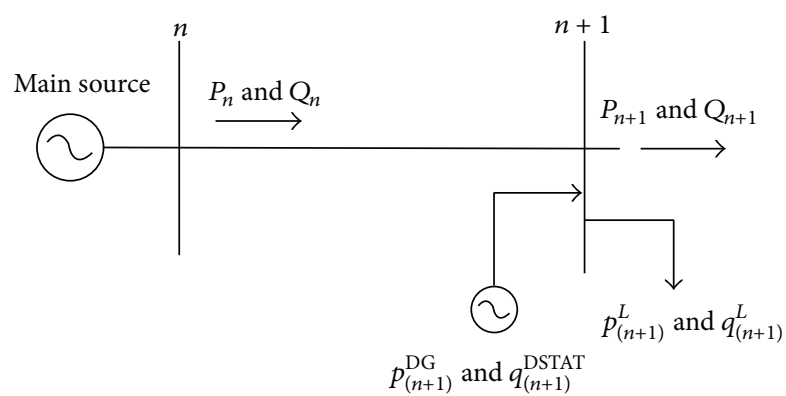

FIGURE 1: Single-line diagram of a two-bus system.

where $\mathrm{PF}_{i}$ is the node voltage deviation penalty factor which is given by

$$
\mathrm{PF}_{i}=\frac{1}{\left(1+\operatorname{Max}\left(\Delta V_{n i}\right)\right)},
$$

where

$$
\begin{aligned}
& \Delta V_{n i} \\
& =\left\{\begin{array}{cc}
1-\left|V_{n i}\right| ; & V_{\min S}<V_{n i}<V_{\min } \\
0 ; & V_{\min } \leq V_{n i} \leq V_{\max } \\
\text { a very large number; } & \text { else }
\end{array}\right\} ; \\
& \forall n \in N, \forall i \in L,
\end{aligned}
$$

subject to the following operational constraints.

(a) Power Flow Equations. The sum of the power purchased from utility grid and the total power generated by the different sources in the distribution system must be balanced by the local load demand and the power loss in the lines. For a radial network, a set of recursive equations are used to model the power flow in the network as shown by (4). A sample twobus system including DG and DSTATCOM units is shown in Figure 1. Consider

$$
\begin{aligned}
& P_{(n+1), i}=P_{n, i}-R_{n} \frac{P_{n, i}^{2}+Q_{n, i}^{2}}{V_{n, i}^{2}}-P_{(n+1), i} ; \\
& \quad \forall n \in N, \forall i \in L, \\
& Q_{(n+1), i}=Q_{n, i}-X_{n} \frac{P_{n, i}^{2}+Q_{n, i}^{2}}{V_{n, i}^{2}}-q_{(n+1), i} ; \\
& V_{(n+1), i}^{2}=V_{n, i}^{2}-2\left(R_{n} P_{n, i}+X_{n} Q_{n, i}\right) \\
& +\left(R_{n}^{2}+X_{n}^{2}\right) \frac{P_{n, i}^{2}+Q_{n, i}^{2}}{V_{n, i}^{2}} ;
\end{aligned}
$$

$\forall n \in N, \forall i \in L$,

$$
\begin{aligned}
& p_{(n+1), i}=p_{(n+1), i}^{L}-p_{(n+1), i}^{\mathrm{DG}} ; \quad \forall n \in N, \quad \forall i \in L, \\
& q_{(n+1), i}=q_{(n+1), i}^{L}-q_{(n+1), i}^{\mathrm{DSTAT}} ; \quad \forall n \in N, \quad \forall i \in L .
\end{aligned}
$$

(b) Branch Current Limit. The current flow in each branch must satisfy the rated ampacity of each branch:

$$
I_{n i} \leq I_{n}^{\max } ; \quad \forall n \in N, \forall i \in L .
$$

(c) Active and Reactive Compensation Limit at a Node. The active and reactive power injected by DG and DSTATCOM at each node must be within their permissible ranges:

$$
\begin{gathered}
p_{n, \text { min }}^{\mathrm{DG}} \leq p_{n}^{\mathrm{DG}} \leq p_{n, \text { max }}^{\mathrm{DG}} ; \quad \forall n \in N, \\
q_{n, \text { min }}^{\mathrm{DSTAT}} \leq q_{n}^{\mathrm{DSTAT}} \leq q_{n, \text { max }}^{\mathrm{DSTAT}} ; \quad \forall n \in N .
\end{gathered}
$$

(d) System Compensation Limit. The sum of active and reactive power injected by DGs and DSTATCOMs at all candidate nodes should be less than system nominal active and reactive power demand, respectively:

$$
\begin{gathered}
\sum q_{n}^{\mathrm{DSTAT}} \leq Q_{D} ; \quad \forall n \in N, \\
\sum p_{n}^{\mathrm{DG}} \leq P_{D} ; \quad \forall n \in N .
\end{gathered}
$$

Equations (8) and (9) ensure nonrepetition of candidate nodes for DSTATCOM and DG allocation, respectively:

$$
\begin{aligned}
N_{\mathrm{DSTAT}, a} & \neq N_{\mathrm{DSTAT}, b} ; \quad a, b \in N, \\
N_{\mathrm{DG}, a} & \neq N_{\mathrm{DG}, b} ; \quad a, b \in N .
\end{aligned}
$$

The distribution network is reconfigured after optimally placing these devices. The reconfiguration problem is solved to minimize real power loss while satisfying various network operational constraints. The mathematical formulation of reconfiguration problem for loss minimization is formulated below:

Minimize, $P_{\text {loss }, i}=\sum_{n=1}^{E} R_{n} \frac{P_{n i}^{2}+Q_{n i}^{2}}{\left|V_{n i}\right|^{2}} ;$

$\forall n \in N, \forall i \in L$,

subject to constraints defined by (4)-(5) along with radial topology and node voltage constraints as given below.

(a) Radiality Constraint. The reconfigured network topology must be radial, that is, with no closed path:

$$
\Phi_{i}(r)=0 ; \quad \forall i \in L .
$$

(b) Voltage Limit Constraint. A hard voltage constraint is employed during the network reconfiguration as it is one of 
the important network operation strategies. All node voltages of the system must be maintained within acceptable operating limits during the optimization process:

$$
V_{\min } \leq V_{n i} \leq V_{\max } ; \quad \forall n \in N, \forall i \in L
$$

In the present work the codification proposed in [18] is used to solve the network reconfiguration problem. This is a rule-based codification to check and correct infeasible radial topologies while attempting this problem.

\section{A Brief Overview of CSO}

Cats initiate their move very slowly and cautiously after sensing the presence of a prey and finally chase it very quickly. By observing these features, two modes of operation for CSO are simulated, that is, seeking and tracing modes. These modes have been mathematically modeled for solving optimization problems and are combined together by defining a mixture ratio (MR). The position of cats represents the set of tentative solutions in the problem search space. Every cat has its own position composed of $D$ dimensions, velocity for each dimension, a fitness value, which represents the accommodation of the cat to the fitness function, and a flag to identify whether the cat is in seeking mode or tracing mode [20]. These two modes of operation can be described briefly as given below.

3.1. Seeking Mode. In this mode, random mutations within narrow range are employed at predefined dimensions on the cats selected according to MR. Some terms related to this mode can be defined as follows: Seeking Memory Pool (SMP) is the number of copies generated for each cat; Seeking Range of Selected Dimension (SRD) is the predefined range of each dimension being selected for mutation; CDC is the Counts of Dimension to Change, that is, the number of dimensions to be mutated. According to MR, a definite number of cats are selected for this mode. Making SMP copies of each cat: randomly select CDC dimensions for each copy. Each of these selected dimensions is varied in the range [-SRD, +SRD]. In this way each cat is mutated to facilitate local search. However, higher SRD $(>2)$ causes unnecessarily larger search space; higher CDC $(>0.6)$ causes much higher diversity of the individuals by mutation, thus delaying the convergence; lower MR $(<0.2)$ causes the cats to spend most of their time resting and observing (seeking mode), thus avoiding local entrapment to suboptimal solutions [26]. The higher the value of SMP $(>4)$ is, the better the exploration will be, but at the cost of more CPU time. Thus in seeking mode, the best values of SRD, CDC, MR, and SMP should be decided by the usual trade-off between accuracy and CPU time of the algorithm.

3.2. Tracing Mode. This mode corresponds to a local search technique for the optimization problem. Cat traces the target while spending high energy in this mode. The rapid chase of the cat is mathematically modeled as a large change in its position. Define position and velocity of $k$ th cat in the $D$ dimensional search space as $X_{k}=\left(X_{k, 1}, X_{k, 2}, \ldots, X_{k, d}\right)$ and
$V_{k}=\left(V_{k, 1}, V_{k, 2}, \ldots, V_{k, d}\right)$. The global best position of the cat swarm is represented as $X_{\text {best }}=\left(X_{\text {best }, 1}, X_{\text {best }, 2}, \ldots, X_{\text {best }, d}\right)$ [22]. In this mode, the cat moves according to its own velocity among all its dimensions so that it can trace the prey, that is, the best fit cat. The velocity $V_{k, d}$ and position $X_{k, d}$ updates of the $k$ th cat are governed by the following relations:

$$
\begin{aligned}
& V_{k+1, d}=V_{k, d}+C \times r(\cdot) \times\left(X_{\text {best }, d}-X_{k, d}\right), \\
& X_{k+1, d}=X_{k, d}+V_{k+1, d},
\end{aligned}
$$

where $C$ is a constant, usually taken as 2 , and $r(\cdot)$ is a random number in the range $[0,1]$. Whether a cat is in seeking or tracing mode, it survives, if its fitness is improved. At the end of each iteration, cats from both seeking and tracing modes merge together before initiating the next iteration.

\section{Proposed ICSO}

In CSO, the seeking mode provides local search whereas the tracing mode searches globally. However, it will be better if the current best cat is allowed to search locally. If it happens, the current best cat may upgrade its fitness, and later on this will positively influence the movement of all the cats going through the tracing mode. Moreover, it can also avoid possible local trappings. Therefore, seeking mode of the standard CSO is modified in the perception of the above facts as described below.

4.1. Modified Seeking Mode. Like other swarm optimization techniques, the philosophy of CSO is "to follow the leader." If the fitness of the current best cat is improved by some means, the convergence of CSO would be improved. It is therefore suggested that the current best cat is mandatorily selected for the seeking mode. Further, the way of generating cats around the current best cat is also different in the proposed seeking mode, as described below.

The operators of CSO inherently generate continuous decision variables. However, the decision variables are strictly integers in this problem. Therefore, while employing local search around the current best cat, two cats are generated from this cat by employing ceiling and flooring of its decision variables. All possible combinations of cats are generated from these two cats. An illustration of modified seeking mode is shown in Figure 2 as a flowchart. For simplicity, it is shown only for two dimensions. The fitness of all these cats is evaluated and the best fit cat is updated, if a better cat is found. However, other cats of the seeking mode are updated as in the standard CSO. The local search around the best cat not only avoids local trappings but also facilitates the exploration of new search points in the problem search space during the tracing mode.

4.2. Intelligent Search. While initializing or otherwise, it will be always better if all the tentative solutions spread in such a way that most of them lie near the promising region. But this is a difficult task. Nevertheless, a sufficient diversity is essential to explore new solution points in the problem search space. Several researchers, as mentioned earlier, have applied 


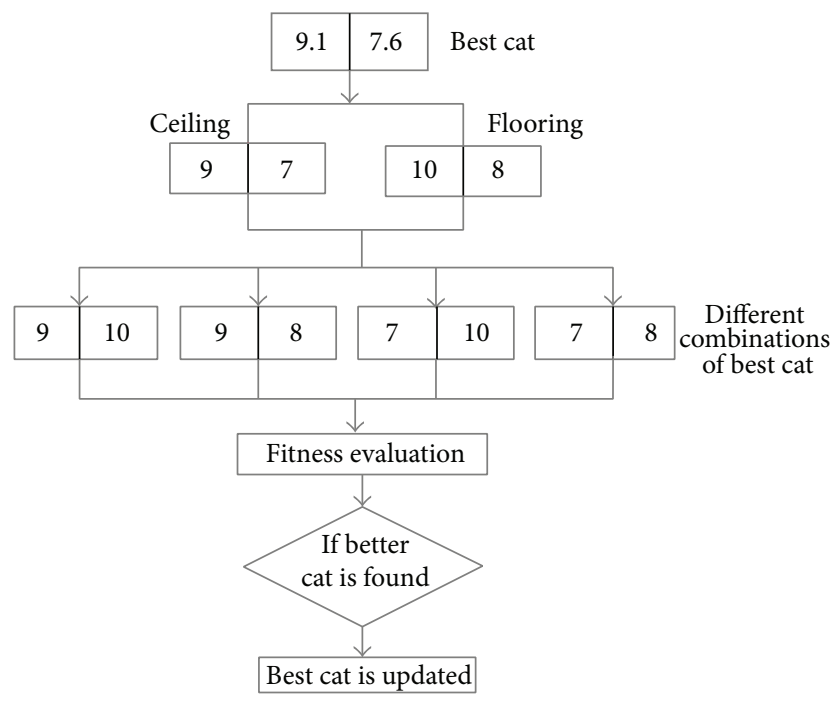

FIGURE 2: Modified seeking mode.

perturbation-based node sensitivity approach to get the node priority list for optimal allocation of distributed resources and then selecting top few nodes from it to redefine the problem search space. However, none of the sensitivity-based approaches is foolproof so it is possible that the optimal node may not lie in the redefined problem search space. Therefore, such approaches are unreliable and may cause erroneous results. In the present work, candidate nodes are selected by proposing an intelligent search approach. The proposed approach is different than the conventional one in the sense that the node sensitivity is observed in terms of the change in objective function to be optimized, instead of the change in power loss. Moreover, the system nodes are arranged in the decreasing order of change in the objective function and then the candidate nodes are selected from this list using roulette wheel selection. In this way, the nodes are selected according to their probability of priority. Thus more chances are available to those nodes which are good for the allocation of these devices. However, this approach reserves the right of each system node to remain in the problem search space during the computational process. Therefore, using intelligent search the problem search space is virtually squeezed without loss of diversity. An illustration of intelligent search is shown in Figure 3.

4.3. Individual's Encoding. The structure of the individuals for the proposed methods is shown in Figure 4 which is composed of candidate nodes and sizing for the respective candidate DSTATCOM and DGs. The candidate nodes are allocated using intelligent search approach, whereas the sizing of distributed resources is selected randomly within their respective predefined bounds as described by (6)-(7).

4.4. Termination Criterion. Elitism is not required due to the intrinsic nature of CSO. Therefore, the termination criterion is taken as follows: "when either the maximum iteration count is exhausted or all cats acquire the same fitness,
TABLE 1: Initial data of 69-bus system.

\begin{tabular}{lc}
\hline Particulars & Value \\
\hline Sectionalizing switches & $1-68$ \\
Base configuration (open lines) & $69-73$ \\
Line voltage (kV) & 12.66 \\
Nominal active demand (kW) & 3802.19 \\
Nominal reactive demand (kVAr) & 2694.6 \\
$P_{\text {loss }}$ at light/nominal/peak load (kW) & $51.61 / 225 / 652.23$ \\
$V_{\min }$ at light/nominal/peak load (p.u.) & $0.9567 / 0.9092 / 0.8445$ \\
\hline
\end{tabular}

TABLE 2: Selected design parameters.

\begin{tabular}{lc}
\hline Parameter & Value \\
\hline loc & 1 \\
$q_{\max }^{\text {DSTAT }}(\mathrm{kVAr}) / p_{\max }^{\mathrm{DG}}(\mathrm{kW})$ & $2000 / 2000$ \\
$Q_{D}(\mathrm{kVAr}) / P_{D}(\mathrm{~kW})$ & $2694.6 / 3802.19$ \\
$N_{\text {DSTAT }} / N_{\mathrm{DG}}$ & $1-69$ \\
$I_{n}^{\max }(n)$ & $400(1-9), 300(46-50,53-65)$, \\
$V_{\min } / V_{\max } / V_{\operatorname{mins}}(\mathrm{p} . u)$. & $200(10-45,51,52,66-73)$ \\
MR/SMP $/$ SRD $/$ CDC & $0.95 / 1.05 / 0.90$ \\
Population size $(P)$ & $0.2 / 3 / 0.2 / 0.6$ \\
Maximum iteration count & 10 \\
\hline
\end{tabular}

TABLE 3: Optimal solution for DSTATCOM and DG allocation.

\begin{tabular}{lcc}
\hline Load levels & $\begin{array}{c}\text { DSTATCOM in kVAr } \\
\text { (node)/DG in kW } \\
\text { (node) }\end{array}$ & Optimal configuration \\
\hline Light & $649(61) / 912(61)$ & $12,20,53,69,72$ \\
Nominal & $1301(61) / 1828(61)$ & $12,21,69,70,72$ \\
Peak & $1998(61) / 1999(61)$ & $12,55,69,70,73$ \\
\hline
\end{tabular}

the evolutionary process stops." This criterion is selected to reduce the CPU time of the algorithm. The flowchart of the proposed ICSO method is presented in Figure 5.

\section{Simulation Results}

The proposed CSO method is investigated on 69-bus test distribution system taken from [30]. The annual load profile is assumed to be piecewise segmented in three different load levels, that is, light, nominal, and peak, which are 50\%, 100\%, and $160 \%$ of the nominal system load, respectively, and the corresponding load durations are taken 2000, 5260, and 1500 hours as in [27]. It has been assumed that DSTATCOM exchanges only reactive power with the network and DG is dispatchable and operated at unity power factor. The initial data of this system are presented in Table 1 and various design parameters selected are given in Table 2 . The NewtonRaphson power flow method is used for load flow of the distribution system.

The optimal solution obtained, for each load level, after 100 trials of proposed ICSO is presented in Table 3. The table shows the capacity of DSTATCOM and DG in kVAr and kW, 


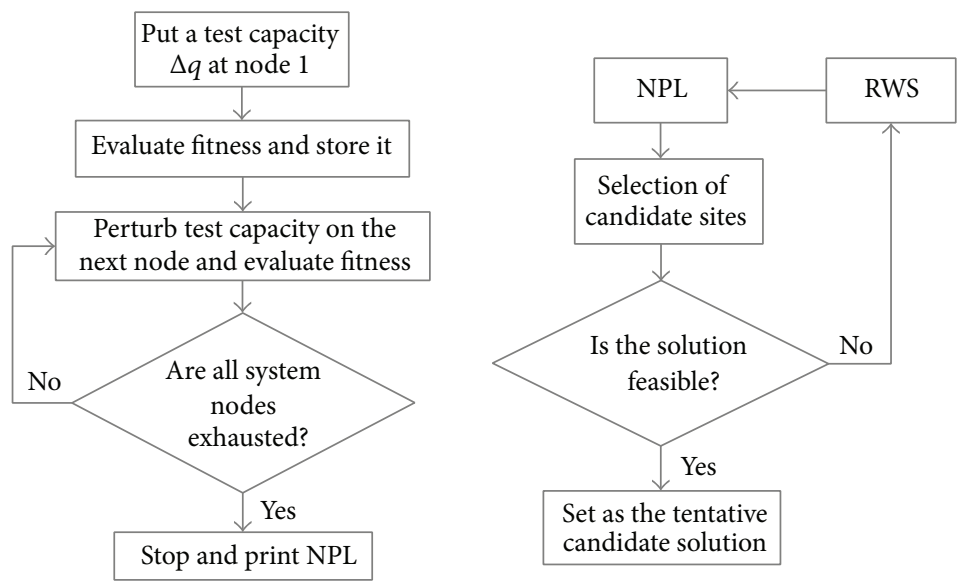

Figure 3: Intelligent search.

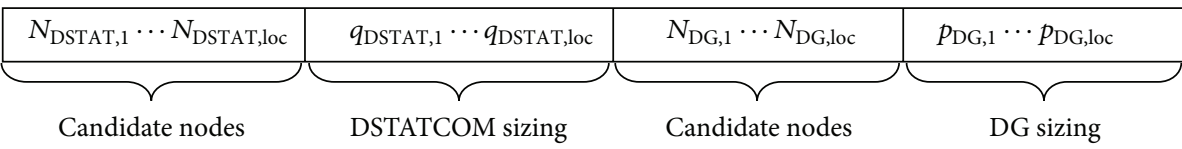

FIgURE 4: Cat's encoding for the proposed ICSO.

respectively. The table also shows that the optimal network configuration is affected by the presence of these devices. The performance of the distribution network obtained using this optimal solution is presented in Table 4.

The table depicts power loss reduction after optimal allocation of these devices for each load level. It can be observed from the table that annual energy losses for this system are reduced significantly by about $88 \%$. This shows that simultaneous placement strategy is very useful for distribution systems. The feeder power losses are further reduced when distribution network is optimally reconfigured after optimally placing DSTATCOM and DG in the distribution system. This causes a net annual energy loss reduction of about $94 \%$ from the base case network which is substantial. Thus, the reconfiguration of distribution network can be fruitfully utilized in order to enhance the energy efficiency of distribution systems. It is interesting to note that the minimum node voltage obtained by optimally placing DSTATCOM and DG is found to be 0.9493 p.u. at peak load level. However, this voltage is enhanced to 0.9751 p.u. by network reconfiguration. This shows the utility of the proposed penalty factor approach where a soft voltage constraint is imposed to permit the solutions having minimum voltage below 0.95 p.u. during evolutionary process.

The voltage profile obtained using the proposed method during all load conditions is presented and compared with base case in Figure 6. It can be observed from the figures that the voltage profile is improved by simultaneous placement of DSTATCOM and DG which is then further enhanced by network reconfiguration. This is true for all load levels considered. All voltages are found to be within permissible limits while integrated approach is employed. Thus the integrated solution provided by the proposed ICSO successfully achieved the desired objectives.
Not much literature is available for the validation of the proposed method. A comparison result with PSO [2] is presented in Table 5. The only scenario is considered where the number of sites for locating each of these components is being restricted to one. The table shows that the power loss reduction and minimum voltage provided by the solution using the proposed method are much better than those obtained with PSO [2]. It happens because the penetration limits of DSTATCOMs and DGs considered in [2] are equal to the nominal load at the nodes where they have to be installed. This penetration limit is 0.888 MVAr and 1.244 MW, respectively, for node 61. However, PSO [2] explores only $0.1223 \mathrm{MW}$ capacities for optimal DGs. Thus, the proposed ICSO is capable of generating a better solution than the existing PSO technique.

\section{Discussion}

The proposed ICSO has shown its potential to efficiently solve one of the large dimension problems of power system. It happens because of the proposed suggestions in the standard CSO. The comparison of the convergence characteristics of CSO and ICSO is presented in Figure 7. The figure also compares the convergence of PSO when applied for the problem with the same population size and maximum iterations. The figure shows a marked improvement in the convergence of ICSO, whereas the CSO and PSO seem to be getting trapped in local optima. It happens due to the poor exploitation of the search space. In ICSO, the intelligent search dispersed all individuals in the near vicinity of the promising region, without scarifying diversity. This causes better chances for good individuals to surf the problem search space amicably. Further, the modified seeking mode enhances 


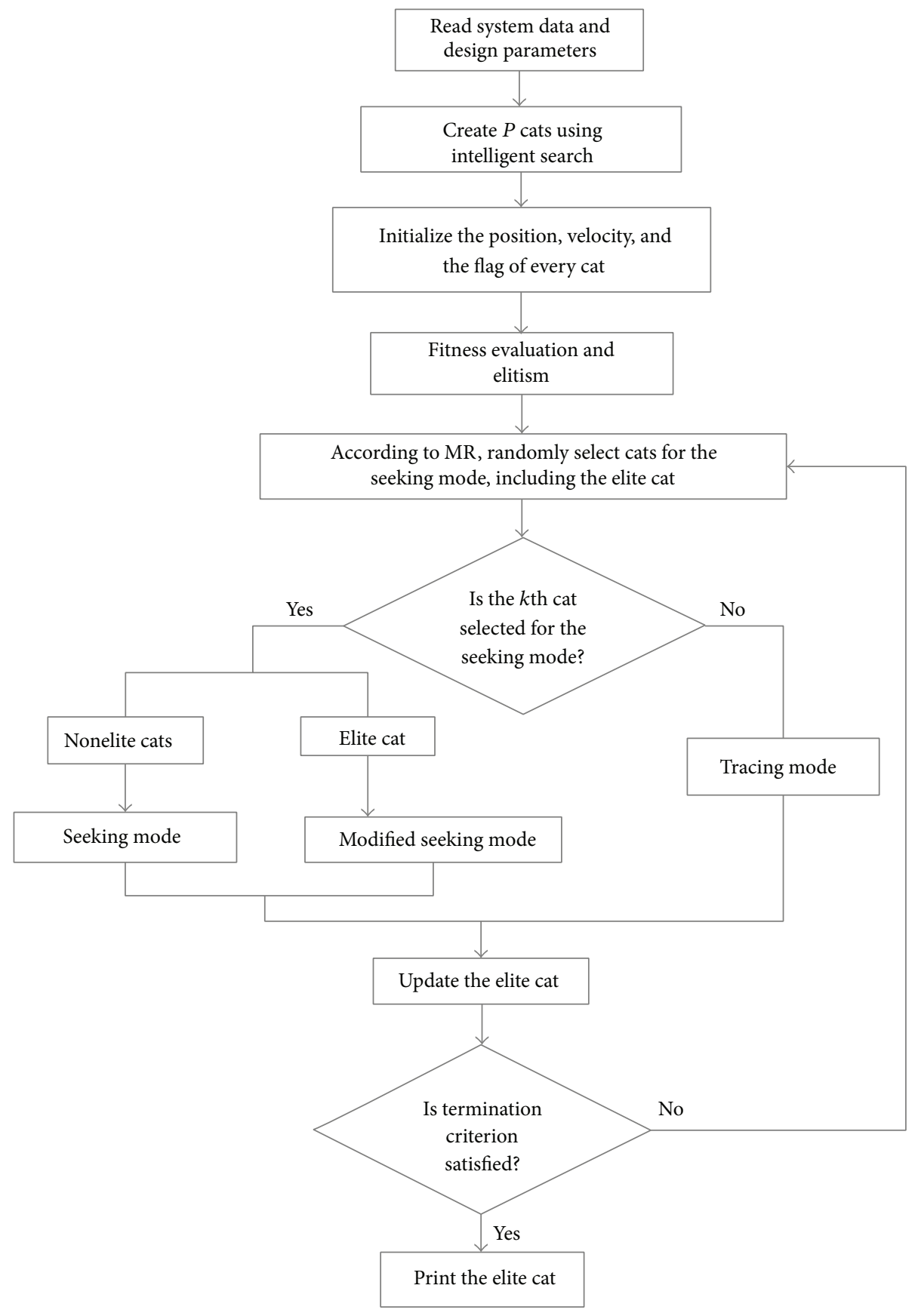

FIGURE 5: Flowchart of the proposed ICSO.

TABLE 4: Distribution network performance using the proposed method.

\begin{tabular}{lccccc}
\hline \multirow{2}{*}{ Particulars } & \multicolumn{3}{c}{ After optimal allocation } & \multicolumn{2}{c}{ Reconfiguration after optimal allocation } \\
& Light & Nominal & Peak & Light & Nominal \\
\hline$P_{\text {loss }}(\mathrm{kW})$ & 5.69 & 23.17 & 92.23 & 2.95 & 12.06 \\
Power loss reduction (\%) & 88.97 & 89.70 & 85.86 & 94.28 & 97.80 \\
$V_{\text {min }}$ (p.u.) & 0.9864 & 0.9725 & 0.9493 & 0.9952 & 92.68 \\
Annual energy loss (kWh) & & 271600 & & & 0.9887 \\
Annual energy loss reduction (\%) & & 88.01 & & & 93.9751 \\
\hline
\end{tabular}




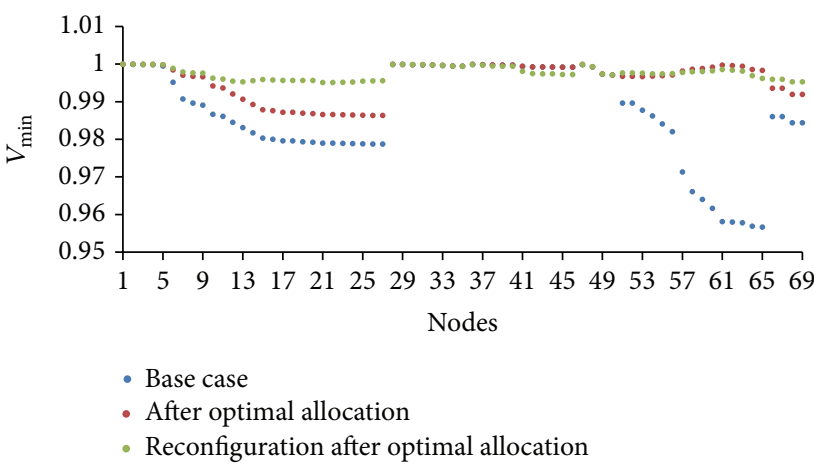

(a)

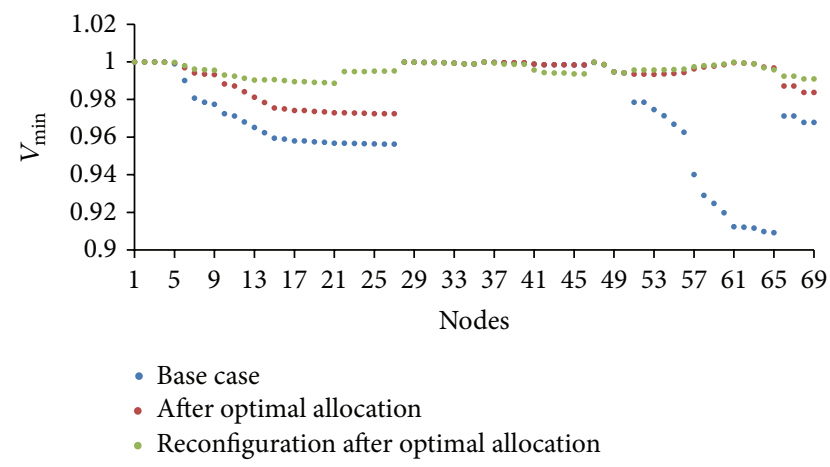

(b)

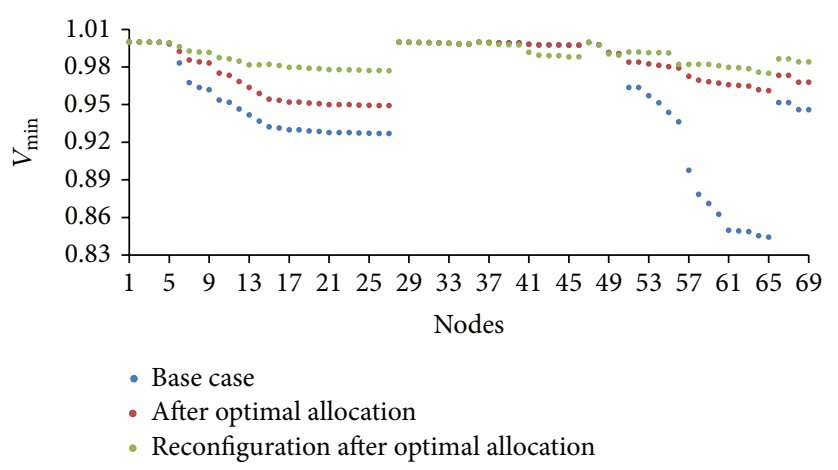

(c)

Figure 6: Comparison of voltage profiles for base case, after optimal allocation of devices and reconfiguration after optimal allocation of devices at (a) light load, (b) nominal load, and (c) peak load.

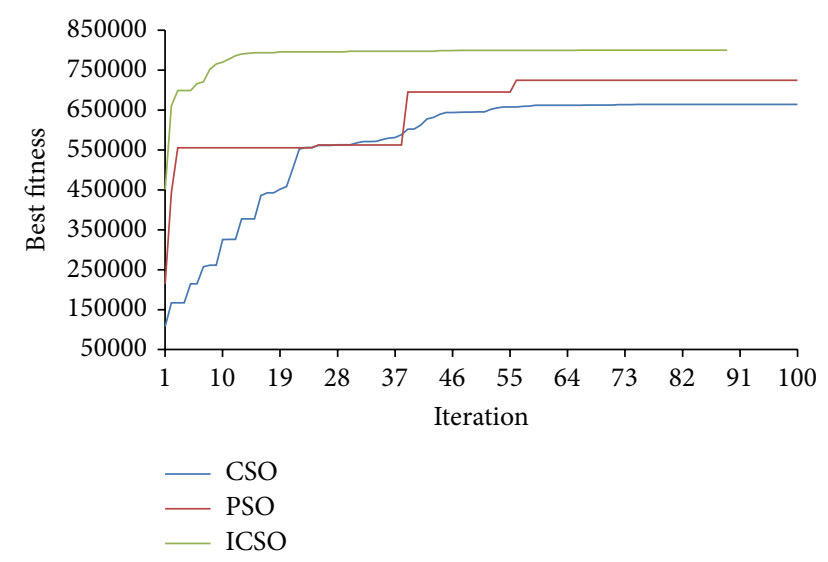

FIGURE 7: Comparison of convergence characteristics of CSO, PSO, and ICSO.

both exploration and exploitation potential of the swarm. This fact can be observed from the typical convergence of ICSO which is quite different than CSO or PSO. As a consequence ICSO facilitates obtaining global or near-global optima in less fitness evaluations.

Finally, a comparison for the solution qualities obtained by CSO, ICSO, and PSO for a sample of 100 independent solutions is presented in Table 6 . The table shows best, mean, and worst fitness of the sampled solutions. It can be observed from the table that the solution quality obtained using ICSO
TABLE 5: Comparison results.

\begin{tabular}{lcc}
\hline Parameters & PSO [2] & ICSO \\
\hline Size of DSTATCOM in MVAr (node) & $0.9045(61)$ & $1.301(61)$ \\
Size of DG in MW (node) & $0.1223(61)$ & $1.828(61)$ \\
$P_{\text {loss }}(\mathrm{kW})$ & 141.14 & 23.17 \\
Power loss reduction (\%) & 37.26 & 89.70 \\
$V_{\text {min }}$ (p.u.) & 0.9291 & 0.9725 \\
\hline
\end{tabular}

is better than either CSO or PSO on the basis of the obtained best, mean, and worst fitness of the sampled solutions. The table also shows statistical indices for the solution quality, that is, standard deviation (SD), coefficient of variation (COV), and error from the best (EFB) of sampled solutions. The smaller the value of these indices, the better the solution quality. It can be said that CSO is performing better than PSO on account of average and best fitness obtained, although it is more computationally demanding. However, ICSO is found to be substantially improved compared to CSO and is less computationally demanding. Therefore, the proposed ICSO not only is capable of generating better solution in less time but also provides good quality solutions. ICSO is taking more time than PSO because the seeking mode of CSO is inherently computationally demanding. 
TABLE 6: Comparison of solution qualities.

\begin{tabular}{lccccccc}
\hline Method & Best & Average & Worst & SD & COV & EFB & CPU time $(\mathrm{s})$ \\
\hline CSO & 783613.33 & 749302.64 & 679973.57 & 30727.05 & 4.10 & 5.88 & 51.87 \\
PSO & 769719.63 & 737032.84 & 705699.83 & 21817.88 & 2.96 & 5.11 & 36.94 \\
Proposed ICSO & 799832.30 & 797367.69 & 778524.72 & 5472.46 & 0.69 & 0.75 & 47.31 \\
\hline
\end{tabular}

\section{Conclusions}

An integrated approach for the optimal placement of distributed resources and network reconfiguration using improved variant of CSO technique is presented. The objectives of maximum power loss reduction and voltage profile enhancements have been successfully achieved using the proposed modeling. It has been observed that the proposed integrated approach of simultaneous placing of DSTATCOMs and DGs in the distribution network and then optimally reconfiguring it can enhance the performance of the systems by a good margin. The suggested seeking mode in ICSO provides local random walk to the best fit cat and the proposed intelligent search scans the problem search space efficiently without loss of diversity. This causes dispersion of all tentative solutions in the close vicinity of the promising region which helps to obtain the global or near-global optima. The comparison result validates that the proposed method is better than existing PSO and CSO methods. It has been observed that the proposed ICSO has better convergence and is also capable of generating fairly good quality solutions.

\section{Nomenclature}

\begin{tabular}{|c|c|}
\hline$C:$ & Acceleration coefficient \\
\hline$D:$ & Number of decision variables \\
\hline$d:$ & $\begin{array}{l}\text { Each dimension of selected cat } \\
(1 \leq d \leq D)\end{array}$ \\
\hline$E:$ & Total number of branches in the system \\
\hline & Feeder current at the $i$ th load level (p.u.) \\
\hline$I_{n}^{\max }:$ & Rated feeder current (p.u.) \\
\hline$L:$ & Set of load levels \\
\hline loc: & $\begin{array}{l}\text { Total number of candidate locations for } \\
\text { DSTATCOM/DG placement }\end{array}$ \\
\hline$N_{\text {DSTAT }}:$ & $\begin{array}{l}\text { Candidate nodes for DSTATCOM } \\
\text { placement }\end{array}$ \\
\hline$N_{\mathrm{DG}}:$ & Candidate nodes for DG placement \\
\hline & Set of system nodes \\
\hline$p_{n, \min }^{\mathrm{DG}} / p_{n, \max }^{\mathrm{DG}}:$ & $\begin{array}{l}\text { Minimum/maximum limits of DG } \\
\text { penetration at the } n \text {th node }(\mathrm{kW})\end{array}$ \\
\hline$p_{n, i}^{\mathrm{DG}}:$ & $\begin{array}{l}\text { DG power generation at the } n \text {th node for } \\
\text { the } i \text { th load level }(\mathrm{kW})\end{array}$ \\
\hline$p_{n, i}^{L} / q_{n, i}^{L}:$ & $\begin{array}{l}\text { Nominal active/reactive power demand at } \\
\text { the } n \text {th node for the } i \text { th load level } \\
(\mathrm{kVAr} / \mathrm{kW})\end{array}$ \\
\hline$P_{D}:$ & $\begin{array}{l}\text { Nominal active power demand of the } \\
\text { system }(\mathrm{kW})\end{array}$ \\
\hline$P_{\text {loss: }}:$ & Power loss $(\mathrm{kW})$ \\
\hline$P_{\text {loss }, b i}:$ & $\begin{array}{l}\text { Power loss for uncompensated system at } \\
\text { the ith load level (kW) }\end{array}$ \\
\hline
\end{tabular}

\begin{tabular}{|c|c|}
\hline$P_{\text {loss }, a i}:$ & $\begin{array}{l}\text { Power loss for compensated system at the } \\
\text { ith load level }(\mathrm{kW})\end{array}$ \\
\hline$P_{n i}:$ & $\begin{array}{l}\text { Real power for sending end of the } n \text {th } \\
\text { branch at the } i \text { th load level }(\mathrm{kW})\end{array}$ \\
\hline$q_{n, i}^{\text {DSTAT }}:$ & $\begin{array}{l}\text { Reactive power injection by DSTATCOM } \\
\text { at the } n \text {th node for the } i \text { th load level } \\
(\mathrm{kVAr})\end{array}$ \\
\hline$q_{n, \min }^{\text {DSTAT }} / q_{n, \max }^{\text {DSTAT }}:$ & $\begin{array}{l}\text { Minimum/maximum reactive } \\
\text { compensation provided by DSTATCOMs } \\
\text { at the } n \text {th node (kVAr) }\end{array}$ \\
\hline$Q_{D}:$ & $\begin{array}{l}\text { Nominal reactive power demand of the } \\
\text { system (kVAr) }\end{array}$ \\
\hline$Q_{n i}:$ & $\begin{array}{l}\text { Reactive power for sending end of the } n \text {th } \\
\text { branch at the } i \text { th load level (kVAr) }\end{array}$ \\
\hline$R_{n}:$ & Line resistance of the $n$th branch $(\Omega)$ \\
\hline$r(\cdot):$ & Random number in the range of $[0,1]$ \\
\hline$V_{\max } / V_{\min }:$ & $\begin{array}{l}\text { Maximum/minimum permissible node } \\
\text { voltage (p.u.) }\end{array}$ \\
\hline & Minimum specified node voltage (p.u.) \\
\hline$V_{n i}:$ & $\begin{array}{l}\text { Voltage of the } n \text {th node at the ith load level } \\
\text { (p.u.) }\end{array}$ \\
\hline$V_{k} / V_{k+1}:$ & Velocity of the $k$ th $/(k+1)$ th cat \\
\hline$\Delta V_{n i}:$ & $\begin{array}{l}\text { Maximum node voltage deviation of the } \\
n \text {th node at the } i \text { th load level (p.u.) }\end{array}$ \\
\hline$X_{\text {best }}:$ & $\begin{array}{l}\text { Position of the cat that has the best fitness } \\
\text { value }\end{array}$ \\
\hline$X_{k} / X_{k+1}:$ & Position of the $k$ th $/(k+1)$ th cat \\
\hline$X_{n}:$ & Line reactance of the $n$th branch $(\Omega)$ \\
\hline & Node voltage deviation penalty factor \\
\hline$\Phi_{i}:$ & Closed loop at the $i$ th load level. \\
\hline
\end{tabular}

\section{Conflict of Interests}

The authors declare that there is no conflict of interests regarding the publication of this paper.

\section{References}

[1] M. E. El-Hawary, "The smart grid-state-of-the-art and future trends," Electric Power Components and Systems, vol. 42, no. 3-4, pp. 239-250, 2014.

[2] S. Devi and M. Geethanjali, "Optimal location and sizing determination of distributed generation and DSTATCOM using particle swarm optimization algorithm," International Journal of Electrical Power \& Energy Systems, vol. 62, pp. 562-570, 2014.

[3] M. A. Darfoun and M. E. El-Hawary, "Multi-objective optimization approach for optimal distributed generation sizing and placement," Electric Power Components and Systems, vol. 43, no. 7, pp. 828-836, 2015.

[4] S. Jazebi, S. H. Hosseinian, and B. Vahidi, "DSTATCOM allocation in distribution networks considering reconfiguration 
using differential evolution algorithm," Energy Conversion and Management, vol. 52, no. 7, pp. 2777-2783, 2011.

[5] S. A. Taher and S. A. Afsari, "Optimal location and sizing of DSTATCOM in distribution systems by immune algorithm," International Journal of Electrical Power \& Energy Systems, vol. 60, pp. 34-44, 2014.

[6] M. Farhoodnea, A. Mohamed, H. Shareef, and H. Zayandehroodi, "Optimum D-STATCOM placement using firefly algorithm for power quality enhancement," in Proceedings of the IEEE 7th International Power Engineering and Optimization Conference (PEOCO '13), pp. 98-102, Langkawi, Malaysia, June 2013.

[7] A. Bagherinasab, M. Zadehbagheri, S. Abdul Khalid, M. Gandomkar, and N. A. Azli, "Optimal placement of D-STATCOM using hybrid genetic and ant colony algorithm to losses reduction," International Journal of Applied Power Engineering, vol. 2, no. 2, pp. 53-60, 2013.

[8] R. Kollu, S. R. Rayapudi, and V. L. N. Sadhu, "A novel method for optimal placement of distributed generation in distribution systems using HSDO," International Transactions on Electrical Energy Systems, vol. 24, no. 4, pp. 547-561, 2014.

[9] J. A. Martín García and A. J. Gil Mena, “Optimal distributed generation location and size using a modified teachinglearning based optimization algorithm," International Journal of Electrical Power and Energy Systems, vol. 50, no. 1, pp. 65-75, 2013.

[10] R. S. Rao, K. Ravindra, K. Satish, and S. V. L. Narasimham, "Power loss minimization in distribution system using network reconfiguration in the presence of distributed generation," IEEE Transactions on Power Systems, vol. 28, no. 1, pp. 317-325, 2013.

[11] S. Kansal, V. Kumar, and B. Tyagi, "Optimal placement of different type of DG sources in distribution networks," International Journal of Electrical Power and Energy Systems, vol. 53, no. 1, pp. 752-760, 2013.

[12] D. K. Khatod, V. Pant, and J. Sharma, "Evolutionary programming based optimal placement of renewable distributed generators," IEEE Transactions on Power Systems, vol. 28, no. 2, pp. 683-695, 2013.

[13] H. Hedayati, S. A. Nabaviniaki, and A. Akbarimajd, "A method for placement of DG units in distribution networks," IEEE Transactions on Power Delivery, vol. 23, no. 3, pp. 1620-1628, 2008.

[14] F. S. Abu-Mouti and M. E. El-Hawary, "Optimal distributed generation allocation and sizing in distribution systems via artificial bee colony algorithm," IEEE Transactions on Power Delivery, vol. 26, no. 4, pp. 2090-2101, 2011.

[15] S. G. Naik, D. K. Khatod, and M. P. Sharma, "Optimal allocation of combined DG and capacitor for real power loss minimization in distribution networks," International Journal of Electrical Power and Energy Systems, vol. 53, pp. 967-973, 2013.

[16] S. M. Sajjadi, M.-R. Haghifam, and J. Salehi, "Simultaneous placement of distributed generation and capacitors in distribution networks considering voltage stability index," International Journal of Electrical Power and Energy Systems, vol. 46, no. 1, pp. 366-375, 2013.

[17] M. H. Moradi, A. Zeinalzadeh, Y. Mohammadi, and M. Abedini, "An efficient hybrid method for solving the optimal sitting and sizing problem of DG and shunt capacitor banks simultaneously based on imperialist competitive algorithm and genetic algorithm," International Journal of Electrical Power and Energy Systems, vol. 54, pp. 101-111, 2014.
[18] A. Swarnkar, N. Gupta, and K. R. Niazi, "A novel codification for meta-heuristic techniques used in distribution network reconfiguration," Electric Power Systems Research, vol. 81, no. 7, pp. 1619-1626, 2011.

[19] M. A. N. Guimarães, C. A. Castro, and R. Romero, "Distribution systems operation optimisation through reconfiguration and capacitor allocation by a dedicated genetic algorithm," IET Generation, Transmission \& Distribution, vol. 4, no. 11, pp. 1213$1222,2010$.

[20] S.-C. Chu and P.-W. Tsai, "Computational intelligence based on the behavior of cats," International Journal of Innovative Computing, Information and Control, vol. 3, no. 1, pp. 163-173, 2007.

[21] S. Temel, N. Unaldi, and O. Kaynak, "On deployment of wireless sensors on 3-D terrains to maximize sensing coverage by utilizing cat swarm optimization with wavelet transform," IEEE Transactions on Systems, Man, and Cybernetics: Systems, vol. 44, no. 1, pp. 111-120, 2014.

[22] P. M. Pradhan and G. Panda, "Solving multiobjective problems using cat swarm optimization," Expert Systems with Applications, vol. 39, no. 3, pp. 2956-2964, 2012.

[23] L. Pappula and D. Ghosh, "Linear antenna array synthesis using cat swarm optimization," AEU-International Journal of Electronics and Communications, vol. 68, no. 6, pp. 540-549, 2014.

[24] G. Panda, P. M. Pradhan, and B. Majhi, "IIR system identification using cat swarm optimization," Expert Systems with Applications, vol. 38, no. 10, pp. 12671-12683, 2011.

[25] Y. Liu, X. Wu, and Y. Shen, "Cat swarm optimization clustering (KSACSOC): a cat swarm optimization clustering algorithm," Scientific Research and Essays, vol. 7, no. 49, pp. 4176-4185, 2012.

[26] S. K. Saha, S. P. Ghoshal, R. Kar, and D. Mandal, "Cat swarm optimization algorithm for optimal linear phase FIR filter design," ISA Transactions, vol. 52, no. 6, pp. 781-794, 2013.

[27] D. Das, "Optimal placement of capacitors in radial distribution system using a Fuzzy-GA method," International Journal of Electrical Power \& Energy Systems, vol. 30, no. 6-7, pp. 361-367, 2008.

[28] R. A. Gallego, A. J. Monticelli, and R. Romero, "Optimal capacitor placement in radial distribution networks," IEEE Transactions on Power Systems, vol. 16, no. 4, pp. 630-637, 2001.

[29] V. Haldar and N. Chakraborty, "Power loss minimization by optimal capacitor placement in radial distribution system using modified cultural algorithm," International Transactions on Electrical Energy Systems, vol. 25, no. 1, pp. 54-71, 2015.

[30] M. E. Baran and F. F. Wu, "Optimal capacitor placement on radial distribution systems," IEEE Transactions on Power Delivery, vol. 4, no. 1, pp. 725-734, 1989. 


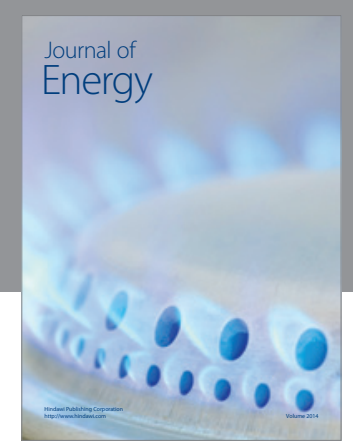

Journal of

Industrial Engineering
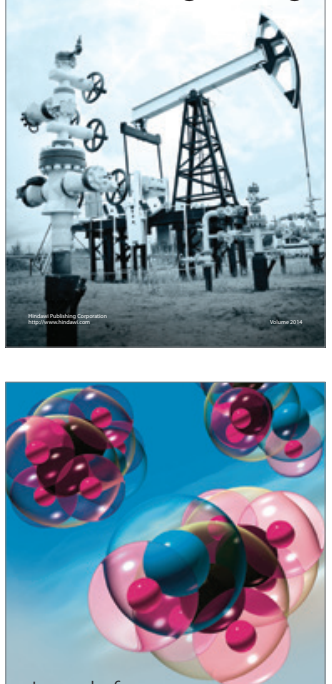

Fuels
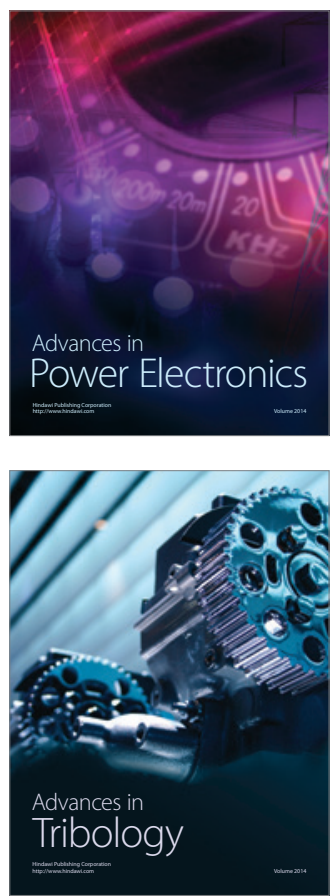

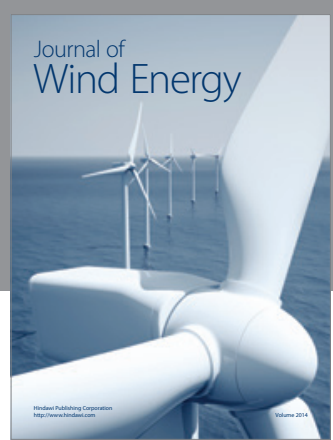

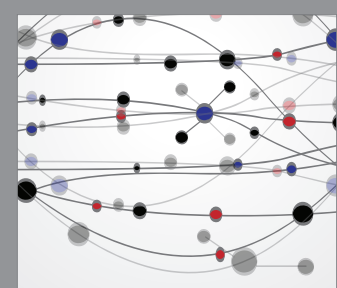

The Scientific World Journal

Submit your manuscripts at http://www.hindawi.com

Journal of

Structures
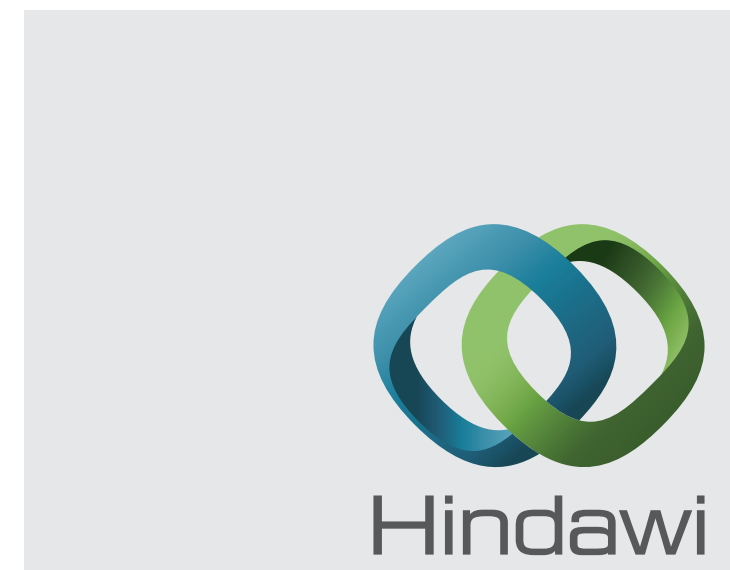

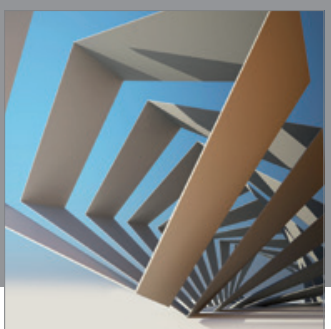

Rotating

Machinery
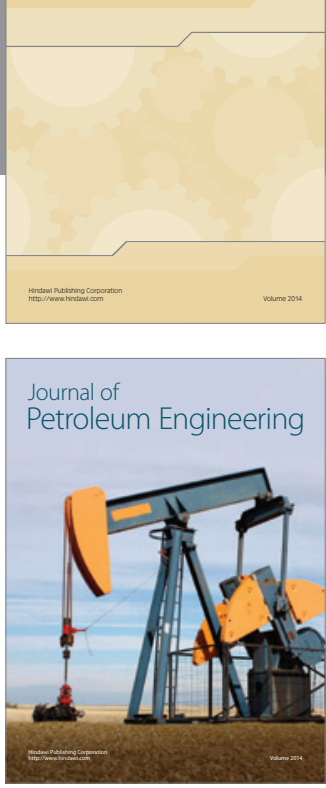

Journal of

Solar Energy
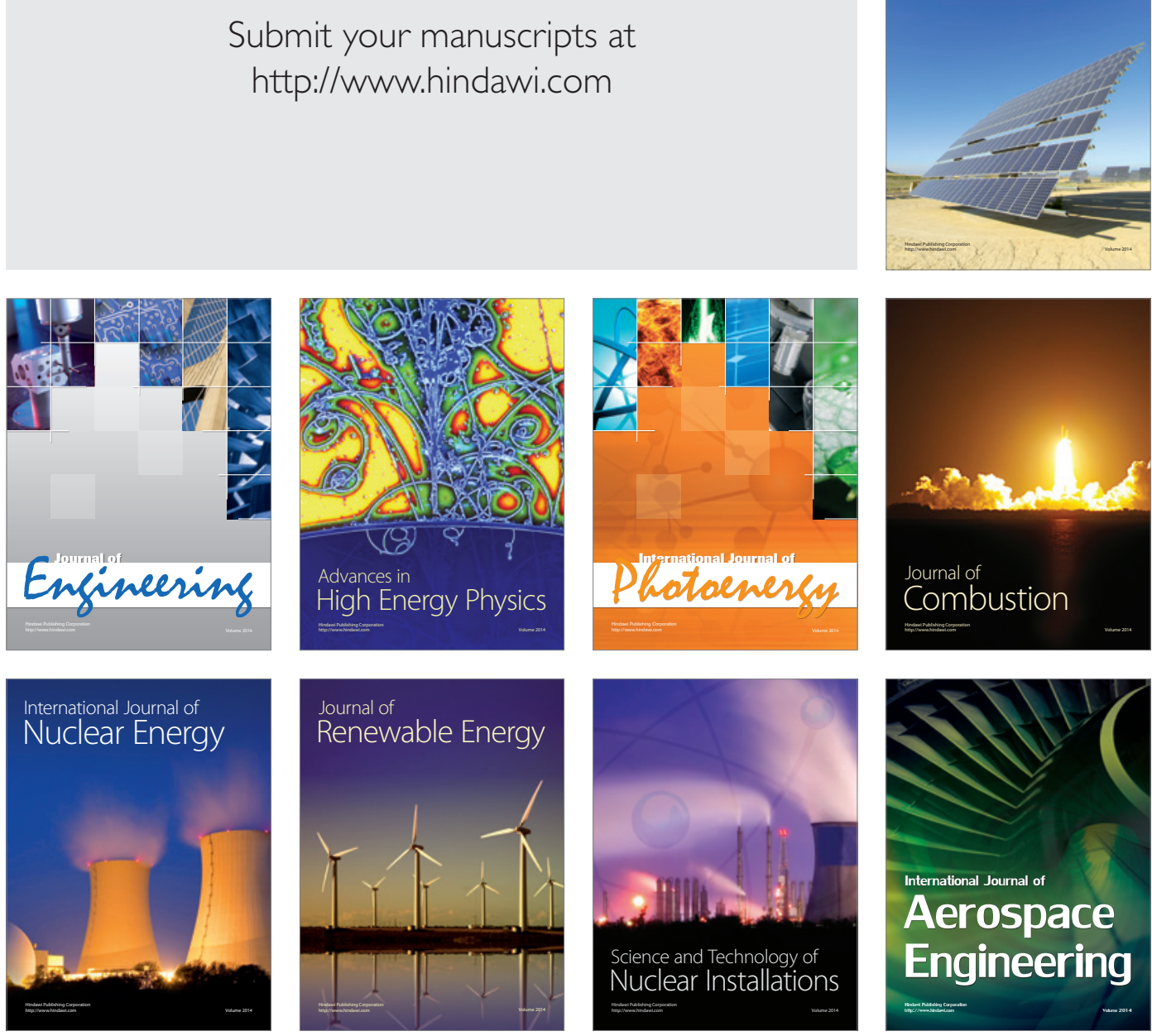